\title{
Lernen als oberste Maxime
}

\section{Liebe Leserinnen und Leser,}

es gibt im Augenblick kaum dynamischere Berufe als den des Controllers - zumindest, wenn man den Berufsverbänden und uns Hochschullehrern glaubt. Kein Stein bleibt auf dem anderen. Der angestammte Platz hinter dem Bildschirm bietet keine Sicherheit mehr. Der Controller muss sich in Management-Themen bewähren. Geschäftskenntnisse sind unverzichtbar geworden. Zugleich nehmen die Anforderungen in den angestammten Expertentätigkeiten zu. Wer nicht aufpasst, dem nimmt Big Data den Status der ,single source of truth“, und ein Business Analyst avanciert schnell zum begehrten Gesprächspartner der Manager - am Controller vorbei.

Controller können einem aktuell fast schon ein wenig leidtun. Nach langen Jahren gefühlt ruhigen Fahrwassers ist die See rau geworden. Man hat den Eindruck, dass sich der schon immer latent geäußerte Verdacht, beim Controller müsse es sich um eine eierlegende Wollmilchsau handeln, heute mit Macht bestätigt. Können Controller wirklich noch dem massiven Erwartungsdruck gerecht werden? Ist das für einen normalen Menschen leistbar?

Wir stellen die Gegenfrage: Warum soll das nicht der Fall sein? Die Praxis scheint uns recht zu geben. Viele CEOs großer Unternehmen waren vor nicht allzu langer Zeit Top-Controller. Wir kennen eine große Zahl von aktuellen Controllern, die ihren Weg in Management-Positionen weitergehen werden, die ihren Controller-Job als eine logische, notwendige Station in ihrer Management-Karriere sehen. Controller, die so angelegt sind, haben in ihrer Entwicklung schon viele Bereiche im Unternehmen kennengelernt, im Inland wie im Ausland. Bei manchen Unternehmen steigt man bewusst so breit ein, wie etwa bei Henkel, wo man in den ersten Jahren zwei Funktionsbereiche, zwei Geschäftsbereiche und zwei Länder kennenlernt. Dies ist eine sehr effektive Form eines systematischen Lernens, für Controller wie für anderen Management-Nachwuchs gleichermaßen. Lernen ist das Schlüsselwort, um den immer höheren Anforderungen gerecht zu werden, Lernen „on the job“ die effektivste Art, dies zu tun.

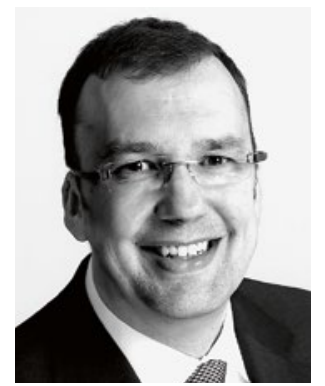

Utz Schäffer

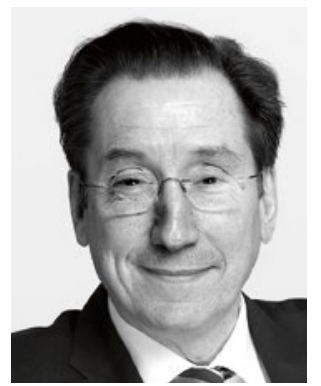

Jürgen Weber
Natürlich reicht das nicht aus. In jeder Funktion - so auch im Controlling - bedarf es einer speziellen fachlichen Weiterbildung. Dies beginnt bei einer klassischen Entsendung zu Spezialseminaren und endet in professionell gestalteten Weiterbildungskonzepten. Neue mediale Lehrformen helfen, den Reiseaufwand zu begrenzen und gleichzeitig Wissen und Ideen quer über das Unternehmen hinweg auszubreiten. Auch eine regelmäßige fachliche Kommunikation via Internet, soziale Medien und periodische Treffen führen dazu, eine Finance Community aufzubauen, Wissen zu distribuieren, kollektiv lernen zu können. Die Finanzperspektive ist für das Unternehmen in allen Management-Fragen wesentlich; sie sollte deshalb von allen Managern beherrscht werden. Auch für diese wird das Anforderungsprofil breiter und umfassender. Nicht nur Controller müssen ständig dazulernen, sondern jeder im erweiterten Management! Wir sind sehr zuversichtlich: Es wird gelingen - auch den Controllern!

Viel Spaß bei der Lektüre wünschen Ihnen

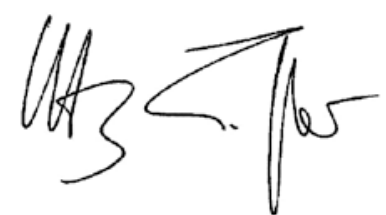

Utz Schäffer

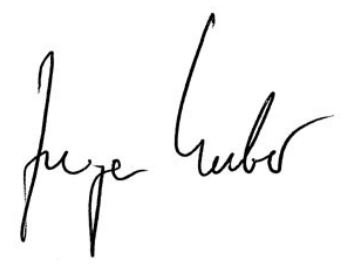

Jürgen Weber 